\author{
Military Technical College \\ Kobry El-Kobbah, \\ Cairo, Egypt
}

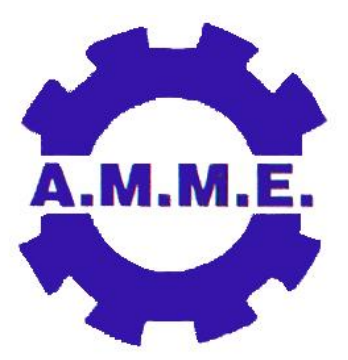

$14^{\text {th }}$ International Conference on Applied Mechanics and Mechanical Engineering.

\title{
BALLISTIC RESISTANCE OF WOVEN FABRIC COMPOSITES
}

\author{
By
}

\author{
M. Abdel-Fattah*, A.M. Riad* and A.I. Fayed*
}

\begin{abstract}
In this paper, the response of woven fabric composites against ballistic impact has been investigated using the analytical model of Ref. [1]. This model is based on energy transfer between the projectile and the target and requires mechanical and fracture properties as well as geometry of target and projectile parameters as input. The main assumptions considered in the analytical model are presented. The equations used for predicting the dissipated energy mechanisms during the ballistic perforation of woven fabric composites are also presented. The governing equations of the model are arranged and compiled into a MATLAB program. The present model predicts the projectile energy loss during the ballistic perforation of woven fabric composites and the corresponding reduction in projectile velocity. The present model is adapted to predict the ballistic resistance of multilayered textile targets.
\end{abstract}

Furthermore, an experimental program has been conducted to study the normal perforation of a $9 \mathrm{~mm}$ projectile into multi-layered textile and woven fabric composites. This program is concerned with the determination of ballistic resistance for a set of targets, consisting of multi-layered twaron and E-glass textiles, twaron/epoxy and Eglass/epoxy composites with different thicknesses. Ballistic measurements are compared with the model predictions; good agreement is generally obtained. The obtained predicted results prove the capability of the analytical model in predicting the response of multilayered textile and woven fabric composite targets, respectively, when impacted by $9 \mathrm{~mm}$ projectile.

KEY WORDS: ballistic impact, woven fabric, wave theory, energy absorbing mechanisms, and residual velocity. 


\section{* Egyptian Armed Forces. INTRODUCTION}

Composite materials are widely used in personal and vehicle armors because of their lightweight and high bulletproof performance. Woven fabric composites have been recognized as more competitive than the unidirectional composites because of their reinforcement in all directions, better toughness and better impact resistance. These properties make the woven fabric composites suitable for body armors. Several penetration models were developed to quantify the penetration resistance of composites. Three different approaches could be followed to design the composite target protections against ballistic impact; these are: (i) analytical, (ii) experimental, and (iii) numerical. Analytical modeling of impact problems in composite materials is the best as it represents a compromise solution between accuracy, cost and physical knowledge. The analytical approach depends on many hypotheses which allow simplifying the solution. It provides the designers by the preliminary composite target defeating a certain threat in few seconds.

Morye, et al. [2] presented a semi-empirical model to simulate the ballistic impact of a 5 $\mathrm{mm}$ steel projectile into nylon-66 fiber composite targets at impact velocity $\left(\mathrm{V}_{\mathrm{i}}\right)$ of $512 \mathrm{~m} / \mathrm{s}$. Their model predicted the effect of fiber modulus, fiber failure strain and energy absorbed in tensile failure of the fiber on the ballistic limit of the composite. The photographs of their experimental program showed a deformation cone due to the impact; complete penetration occurred when the cone radius reached a value of $13.8 \mathrm{~mm}$ and the projectile exit with a residual velocity of $191 \mathrm{~m} / \mathrm{s}$. Moreover, they found no evidence for fiber residual stretching, suggesting that the material was highly elastic and its failure always occurred after the elastic limit.

Vinson and Zukas [3] developed a model determining the actual response of textile fabric panels subjected to ballistic impact by a dense projectile. They formulated stepwise procedures for calculating strains, projectile position, forces and decelerations as functions of penetration time. Their analytical results were in good agreement with experimental data due to the impact of a $5.6 \mathrm{~mm}$ projectile into 1 and 12 plies of nylon and 24 plies of kevlar-29 textile, respectively. Taylor and Vinson [4] extended the model of Ref. [3] by determining the material properties of a target and the geometry of a deflected cone at each time step, allowing a complete description of the impact event. They also performed ballistic tests by impacting 5.6 and $9 \mathrm{~mm}$ bullets against single and multilayered Kevlar-29 fabrics, respectively, to assess their model predictions.

Zhu, et al. [5] developed an analytical model describing the normal impact and perforation of a conical- tipped hard steel cylinder into kevlar-29/polyster composite laminates. They modeled the dissipative mechanisms including indentation of striker tip, bulging at the back surface, fiber failure, delamination and friction using simplified assumptions. They divided the impact event into three consecutive phases; these were indentation, perforation and exit phases. They also performed an experimental program in which they measured the ballistic limit and projectile velocity after perforating the tested laminates. Good agreement was obtained between their model predictions and experimental measurements. 
Gu [6] developed an analytical model to predict the decrease in kinetic energy and residual velocity of a projectile after perforating targets consisting of multi-layered plainwoven fabrics. He ignored the deformation of projectile and the heat generated at the projectile-target interface. He applied the energy conservation principle considering that the kinetic energy loss of the projectile was only equal to the kinetic energy and strain energy of the plain fabric in the impact-deformed region. He also considered the high strain rate of the fibers in fabric when perforated by a high velocity projectile. Predictions of residual velocities and energy absorbed by the multi-layered woven fabrics gave good agreement with the corresponding measurements of his experimental program.

Castillo, et al. [7] studied analytically and numerically the effect of a biaxial preload on the behavior of glass/polyester woven-laminate plates subjected to high-velocity impact. They introduced an analytical model based on energy conservation principle considering the presence of in-plane preload. The results of their analytical model for the biaxial preload state were compared with those found for a non-preload plate. They performed experimental tests to assess the predictions of their analytical model and numerical simulation. Both the predictions of their model and numerical simulation gave good agreement with their experimental measurements.

The analytical models developed by Smith, et al. [8] and Roylance, et al. [9] for describing the impact in yarns and textiles, respectively, were used with a new failure criterion of yarns to build a simple analytical model describing the ballistic impact in textiles [10]. This model has been validated using Dyneema armors and predicts the residual velocities of FSPs (Fragment Simulating Projectiles), with masses ranging from 0.2 to $10 \mathrm{~g}$ and impact velocities ranging from 300 to $1500 \mathrm{~m} / \mathrm{s}$. The model has been provided with a delamination equation in order to include the composite characteristics of delamination. The model was based on the one applied to yarns and afterwards modeling was extended to a fabric and finally to a composite. The experimental measurements gave good agreement with predictions of the analytical model when Dyneema fabric armors were considered.

In the following, the analytical model of Ref. [1] is selected to evaluate the ballistic resistance of woven fabric composites when impacted by small caliber projectiles. The selected model is based on energy transfer between the projectile and the target and requires mechanical and fracture properties as well as geometry of target and projectile parameters as input. Main assumptions considered in the analytical model are introduced and its governing equations are presented. The model predicts the projectile energy loss during the ballistic perforation of woven fabric composite targets and the corresponding reduction in projectile velocity. The present model is also adapted to predict the ballistic resistance of multi-layered textile targets. Moreover, an experimental program has been conducted to assess the model predictions. Representative samples of experimental measurements and corresponding predictions of the analytical model are presented with relevant analyses and discussions.

\section{ANALYTICAL MODEL}

The selected analytical model that describes the normal perforation of woven fabric composites by a small caliber projectile is presented herein [1]. This model is based on energy transfer between the projectile and the target. On striking a target, energy of the projectile is absorbed by various mechanisms like kinetic energy of moving cone $E_{\mathrm{KE}}$, 
shear plugging $E_{S P}$, deformation of secondary yarns $E_{D}$, tension in primary yarns $E_{T F}$, delamination $E_{D L}$, matrix cracking $E_{M C}$, frictional energy $E_{F}$ along with other dissipation mechanisms.

Cone formation was observed on the back face of the target as shown in Fig. 1 when struck by a projectile [2]. Shear formation on the front face and cone formation on the back face start taking place depending on target material properties during the ballistic impact. Initially, the moving cone has velocity equal to that of the projectile and has zero mass. As time progresses, the mass of moving cone increases and its velocity decreases. As the cone formation takes place, the yarns/fibers deform and absorb some energy. The primary yarns, which provide the resistive force to the projectile motion, are strained the most, thus leading to their failure. When all primary yarns fail, the projectile exits the target. Tensile failure of the yarns thus absorb some energy of the projectile. Even before the failure of primary yarns, there would be some energy absorption because of tension in the yarns. During the ballistic impact, delamination and matrix cracking take place in the laminate area of composite targets, which forms the cone.

In the following, the analytical model describing the normal perforation of a woven fabric composites by a small caliber projectile is presented. Firstly, modeling of a single yarn is presented. The model is further extended to woven fabric composite targets.

\section{Main Assumptions}

- Projectile is perfectly rigid and remains undeformed during the ballistic impact.

- Projectile motion is uniform during penetration within each time interval.

- Energy absorption is due to primary yarns/fibers breakage and deformation of the secondary yarns are treated independently

- Longitudinal and transversal wave velocities are the same in all the layers.

- Energy absorbed due to friction between projectile and composite is neglected.

- The distance traveled by the projectile and the depth of the cone formed are equal, and the velocities of the projectile and the moving cone would be equal.

- For multi-layered textile targets, energies absorbed due to delamination and matrix cracking are neglected.

- The change of strain with thickness is considered to be linear.

\section{Modeling a Single Yarn subjected to Ballistic Impact}

when a yarn is impacted by a projectile, a longitudinal strain wavelet called the plastic wave is initiated and propagates horizontally with velocity " $\mathrm{C}_{\mathrm{p}}$ " along the yarns. As the wave passes a given point on the yarn, material of the filament flows inward towards the impact point. The material in the wake of the plastic wave front forms itself into a transverse wave, shaped like an inverted tent with the impact point at its vertex, as shown in Fig. 2. The transverse wave propagates with velocity " $\mathrm{C}_{t}$ ". The transverse and longitudinal wave velocities were determined using Taylor and Vinson's model [4].

The transverse wave velocity is given by:

$$
c_{t}=74+(0.64 \cdot V) \text {, }
$$


where " $V$ " is the projectile velocity.

The longitudinal (plastic) wave velocity as function of the strain in yarn, $\varepsilon$, and the transverse wave velocity, $c_{t}$, is given by:

$$
c_{p}=\frac{c_{t}}{\left\{[\varepsilon(1+\varepsilon)]^{1 / 2}-\varepsilon\right\}} .
$$

If the impact event is divided into a number of small instants, then at $i^{\text {th }}$ instant, the time is given by $t_{i}$. By that time, the transverse wave has traveled to a distance " $r_{t i}$ " and the plastic wave has traveled to a distance " $r_{p i}$ ". The projectile has moved through a distance "zi".

Radii $r_{t i}$ and $r_{p i}$ after time $t i=i \Delta t$ are given by:

$$
\mathrm{r}_{\mathrm{ti}}=\sum_{\mathrm{n}=0}^{\mathrm{n}=\mathrm{i}} \mathrm{c}_{\mathrm{tn}} \Delta \mathrm{t}
$$

and

$$
\mathrm{r}_{\mathrm{pi}}=\sum_{\mathrm{n}=0}^{\mathrm{n}=\mathrm{i}} \mathrm{c}_{\mathrm{pn}} \Delta \mathrm{t}
$$

\section{Modeling Woven Fabric composites}

Woven fabric composites consist of warp and fill yarns, interlaced in a regular sequence. As the projectile impacts into a woven fabric composite, there can be many yarns beneath the projectile. In the present analysis, all the warp and fill yarns are treated separately. Behavior of each yarn is analyzed as explained previously. Overall behavior of a woven fabric composite is presented considering the combined effect of all individual yarns within a layer.

\section{Strain and Deceleration History: Single Yarn of a Woven Fabric Composite}

When a projectile impacts a woven fabric composite, a cone would be formed on the back face of the target. The cone formed is considered to have a circular cross-section with a diameter $d$. The base of the cone spreads with a transverse wave velocity. After time $t_{i}$, the transverse wave travels to a distance $r_{\mathrm{ti}}$ and the plastic wave travels to a distance $r_{\mathrm{pi}}$, Cf. Eqns. (3) and (4). Because of stress wave attenuation, stress in the yarn, and hence strain in the yarn, varies with the distance from the point of impact. Stress and, in turn, strain is maximum at the point of impact and decreases along the length of the yarn. At time $t_{i}$, the strain at the point of impact, $\varepsilon_{i}$, is given by [1]:

$$
\varepsilon_{\mathrm{i}}=\left(\frac{(\mathrm{d} / 2)+\sqrt{\left(\mathrm{r}_{\mathrm{ti}}-(\mathrm{d} / 2)\right)^{2}+\mathrm{z}_{\mathrm{i}}^{2}}-\mathrm{r}_{\mathrm{ti}}}{\mathrm{b}^{\left(\mathrm{r}_{\mathrm{pi}} / \mathrm{a}\right)}-1}\right) \cdot\left(\frac{\ln \mathrm{b}}{\mathrm{a}}\right),
$$

where $d$ is the projectile diameter, $b$ is a stress wave attenuation factor and a is the yarn width. The factor $b$ depends on geometry of the fabric as well as mechanical and physical 
properties of the reinforcing material and matrix. For a woven fabric composite, $b=0.825$ [11].

At the beginning of the first time interval of impact, the entire energy is in the form of kinetic energy of projectile. Later, this energy is dissipated into energies absorbed by various damage mechanisms and the kinetic energies of moving cone and projectile, respectively. Considering the energy balance at the end of the $i^{\text {th }}$ time interval, the initial kinetic energy of projectile is distributed as [1]:

$$
\begin{gathered}
\mathrm{KE}_{\mathrm{Po}}=\mathrm{KE}_{\mathrm{Pi}}+\mathrm{E}_{\mathrm{KE}}+\mathrm{E}_{\mathrm{SP}(\mathrm{i}-1)}+\mathrm{E}_{\mathrm{D}(\mathrm{i}-1)}+\mathrm{E}_{\mathrm{TF}(\mathrm{i}-1)}+\mathrm{E}_{\mathrm{DL}(\mathrm{i}-1)}+ \\
\mathrm{E}_{\mathrm{MC}(\mathrm{i}-1)}+\mathrm{E}_{\mathrm{F}(\mathrm{i}-1)},
\end{gathered}
$$

where " $K E_{p o}$ " is the initial kinetic energy of the projectile, "KE$p E_{p i}$ is the kinetic energy of the projectile at time $t_{i}, " E_{K E}$ " is the kinetic energy of the moving cone at time " $t_{i}$, and $E_{s p(\mathrm{i}-1)}, E_{D(\mathrm{i}-1)}, E_{T F(\mathrm{i}-1)}, E_{D L(\mathrm{i}-1)}, E_{M C(\mathrm{i}-1)}$, and $E_{F(\mathrm{i}-1)}$ are the energies absorbed by shear plugging, deformation of secondary yarns, tensile failure of primary yarns, delamination, matrix cracking, and friction till time $\mathrm{t}_{(\mathrm{i}-1)}$, respectively.

Rearranging the terms in the above equation, the following is obtained:

$$
\frac{1}{2} \mathrm{~m}_{\mathrm{p}} \mathrm{V}_{0}^{2}-\mathrm{E}_{(\mathrm{i}-1)}=\frac{1}{2}\left(\mathrm{~m}_{\mathrm{p}}+\mathrm{M}_{\mathrm{Ci}}\right) \mathrm{V}_{\mathrm{i}}^{2}
$$

where " $m_{p}$ " is the mass of the projectile, " $V_{o}$ " is the projectile impact velocity, " $V_{i}$ " is the projectile velocity at time " $t_{i}$ ", and " $M_{c i}$ " is the mass of the cone at time " $t_{i}$ ". The term " $E_{(i-1)}$ " is given by:

$$
\mathrm{E}_{(\mathrm{i}-1)}=\mathrm{E}_{\mathrm{SP}(\mathrm{i}-1)}+\mathrm{E}_{\mathrm{D}(\mathrm{i}-1)}+\mathrm{E}_{\mathrm{TF}(\mathrm{i}-1)}+\mathrm{E}_{\mathrm{DL}(\mathrm{i}-1)}+\mathrm{E}_{\mathrm{MC}(\mathrm{i}-1)}+\mathrm{E}_{\mathrm{F}(\mathrm{i}-1)} .
$$

The terms on the right hand side of Eqn. (8) are known at (i-1)th instant of time. From this, the velocity of the projectile at the end of the $\mathrm{i}^{\text {th }}$ time interval can be obtained as:

$$
V_{i}=\sqrt{\frac{\frac{1}{2} m_{p} V_{o}^{2}-E_{i-1}}{\frac{1}{2}\left(m_{p}+M_{C_{i}}\right)}}
$$

If the projectile velocity is known at the beginning and the end of the $i^{\text {th }}$ time interval, the deceleration of the projectile during that time interval can be found as:

$$
\mathrm{dc}_{\mathrm{i}}=\frac{\mathrm{V}_{\mathrm{i}-1}-\mathrm{V}_{\mathrm{i}}}{\Delta \mathrm{t}}
$$

Utilizing " $d c_{i}$ ", the force resisting the projectile motion can be calculated by:

$$
F_{i}=m_{p} \cdot d c_{i}
$$


It may be noted that the velocity at the beginning of the $i^{\text {th }}$ time interval is the same as the velocity at the end of the $(\mathrm{i}-1)^{\text {th }}$ time interval. The distance traveled by the projectile "zi" up to the $\mathrm{i}^{\text {th }}$ time interval is given by [1]:

$$
\mathrm{z}_{\mathrm{i}}=\sum_{\mathrm{n}=0}^{\mathrm{n}=\mathrm{i}} \Delta \mathrm{z}_{\mathrm{n}},
$$

where

$$
\Delta \mathrm{z}_{\mathrm{i}}=\mathrm{V}_{\mathrm{i}-1} \Delta \mathrm{t}-\frac{1}{2} \mathrm{dc}_{\mathrm{i}} \Delta \mathrm{t}^{2}
$$

Once, the strain variation in a yarn/fibre is known, the energy absorbed by various mechanisms can be calculated, Cf. Eqn. (5). The above process is repeated until all the primary yarns in the target fail, i.e., the complete perforation takes place. The velocity at the end of the time interval, during which all the yarns are broken, is the residual velocity of the projectile. If the exit velocity is zero, the corresponding impact velocity of the projectile is the ballistic limit. If the projectile velocity is zero during the contact event before all the fibres are broken, it indicates that only partial penetration has occurred. Then the projectile does not penetrate the target completely with the given impact velocity. Thus by repetition of the above procedure with various velocities so as to get complete perforation with zero residual velocity, the ballistic limit of the target laminate can be obtained.

\section{Kinetic Energy of the Moving Cone Formed}

The cone formed on the back face of the target absorbs some energy. By the end of the $\mathrm{i}^{\text {th }}$ time interval, the surface radius of the cone formed is given by Eqn. (3). Mass of the cone formed is:

$$
M_{C_{i}}=\pi \cdot r_{t_{i}}^{2} \cdot h \cdot \rho
$$

where $\mathrm{h}$ is the target thickness and $\rho$ is the density of the target.

The velocity of the cone formed is equal to the velocity of the projectile at the end of the $i^{\text {th }}$ time interval, $V_{i}$. So the energy of the cone formed at the end of the $i^{\text {th }}$ time interval is given by:

$$
\mathrm{E}_{\mathrm{KEi}}=\frac{1}{2} \mathrm{M}_{\mathrm{C}_{\mathrm{i}}} \mathrm{V}_{\mathrm{i}}^{2}
$$

where " $M_{c i}$ " is the mass of the cone at time " $t_{i}$.

\section{Energy Absorbed due to Shear Plugging}

When the target material is impacted by the projectile, shear plugging stress in the material near the projectile periphery rises. When the shear plugging stress exceeds shear plugging strength, shear plugging failure occurs. As a result, plug formation takes place. At the beginning of the time interval $t_{i}$, if shear plugging exceeds shear plugging strength, the energy absorbed by shear plugging during this time interval is given by [1]: 


$$
\Delta \mathrm{E}_{\mathrm{SPi}}=\mathrm{Nh}_{1} \mathrm{~S}_{\mathrm{SP}} \pi \mathrm{dh}
$$

where $N$ is the number of layers shear-plugged during the $i^{\text {th }}$ time interval, $h_{l}$ is the thickness of layer, and $S_{s p}$ is the shear plugging strength.

The energy absorbed by shear plugging at the end of the $\mathrm{i}^{\text {th }}$ time interval is given by:

$$
\mathrm{E}_{\mathrm{SPi}}=\sum_{\mathrm{n}=1}^{\mathrm{n}=\mathrm{i}} \Delta \mathrm{E}_{\mathrm{SPn}} .
$$

\section{Energy Absorbed due to Deformation of Secondary Yarns}

The secondary yarns experience different strains depending on their position. The yarns, which are close to the point of impact experience a strain equal to the strain in the outermost primary yarns, whereas the yarns away from the impact point experience less strain.

The energy absorbed in the deformation of all the secondary yarns can be obtained by the following integration [1]:

$$
\mathrm{E}_{\mathrm{D}_{\mathrm{i}}}=\int_{\mathrm{d} / 2}^{\mathrm{r}_{\mathrm{ti}}}\left(\int_{0}^{\varepsilon_{\mathrm{sy}}} \sigma_{\mathrm{sy}}\left(\varepsilon_{\mathrm{sy}}\right) \mathrm{d} \varepsilon_{\mathrm{sy}}\right) \mathrm{h}\left[2 \pi \mathrm{r}-8 \mathrm{r} \sin ^{-1}\left(\frac{\mathrm{d}}{2 \mathrm{r}}\right)\right] \mathrm{dr},
$$

where $\varepsilon_{\text {sy }}$ is the strain in secondary yarns, and $\sigma_{\text {sy }}$ is the stress in such yarns.

The strain variation from $A$ to $B$ is assumed to be linear, Cf. Fig. 1. This imposes the following boundary conditions for the variation in strain at time $t_{i}$ :

$$
\begin{array}{ccc}
\varepsilon_{s y}=\varepsilon_{p y} \quad \text { at } & \mathrm{r}=\frac{\mathrm{d}}{\sqrt{2}} \quad \text { at point A. } \\
\varepsilon_{s y}=0 \quad \text { at } \quad \mathrm{r}=\mathrm{r}_{\mathrm{ti}} & \text { at point } \mathrm{B} .
\end{array}
$$

The linear strain variation from $A$ to $B$ can be expressed by [1]:

$$
\varepsilon_{\mathrm{sy}_{\mathrm{i}}}=\frac{\sqrt{2}\left(\mathrm{r}_{\mathrm{ti}}-\mathrm{r}\right)}{\left(\sqrt{2} \mathrm{r}_{\mathrm{ti}}-\mathrm{d}\right)} \varepsilon_{\mathrm{pyi}} .
$$

\section{Energy Absorbed due to Tension in Primary Yarns}

These primary yarns below the projectile fail by direct tension. All the primary yarns within one layer do not fail at the same instant. When the strain of a particular yarn reaches the dynamic failure strain in tension, the yarn fails. The energy absorbed due to tensile failure of yarn/fiber having a cross-sectional area $A$ is given by [1]: 


$$
E_{T F_{i}}=A \int_{0}^{x=r_{p_{i}}}\left(\int_{\varepsilon=0}^{\varepsilon=\varepsilon_{0} b^{x / a}} \sigma(\varepsilon) d \varepsilon\right) d x
$$

where $\varepsilon_{o}$ is the ultimate strain in yarn/fiber. If during the $\mathrm{i}^{\text {th }}$ time interval, $\mathrm{N}$ numbers of yarns/fibers fail, then the right hand side of Eqn. (21) is multiplied by $\mathrm{N}$.

\section{Energy Absorbed due to Delamination and Matrix Cracking}

Delamination and matrix cracking absorb some part of the initial kinetic energy of the projectile. The area undergoing delamination and matrix cracking in the conical region is of quasi-lemniscate shape (Cf. Fig. 3), which is taken to be $A_{q l}$ percent of the corresponding circular area. During the $i^{\text {th }}$ time interval, the area of delamination and matrix cracking is given by [12]:

$$
\pi\left(r_{d i}^{2}-r_{d(i-1)}^{2}\right) A_{q l}
$$

where $r_{d(i-1)}$ is the radius up to which the damage has propagated until the $i^{\text {th }}$ time interval. So the respective energies absorbed by delamination and matrix cracking during this time interval are given by:

$$
\Delta \mathrm{E}_{\mathrm{DLi}}=\mathrm{P}_{\mathrm{d}} \cdot \pi \cdot\left(\mathrm{r}_{\mathrm{di}}^{2}-\mathrm{r}_{\mathrm{d}(\mathrm{i}-1)}^{2}\right) \cdot \mathrm{A}_{\mathrm{ql}} \cdot \mathrm{G}_{\mathrm{IIed}}
$$

and

$$
\Delta \mathrm{E}_{\mathrm{MCi}}=\mathrm{P}_{\mathrm{m}} \cdot \pi \cdot\left(\mathrm{r}_{\mathrm{di}}^{2}-\mathrm{r}_{\mathrm{d}(\mathrm{i}-1)}^{2}\right) \cdot \mathrm{A}_{\mathrm{ql}} \cdot \mathrm{E}_{\mathrm{mt}} \cdot \mathrm{h} \cdot \mathrm{V}_{\mathrm{m}}
$$

where $G_{\text {Iled }}$ is the critical dynamic strain energy release rate in mode II, $P_{d}$ and $P_{m}$ are percentage of delamination and matrix cracking, $E_{m t}$ is the energy absorbed by matrix cracking per unit volume, $V_{m}$ is the matrix volume fraction and $A q /$ is quasi-lemniscate area reduction factor.

Energy absorbed due to delamination and matrix cracking until the $\mathrm{i}^{\text {th }}$ time interval is given by [1]:

$$
\mathrm{E}_{\mathrm{DLi}}=\sum_{\mathrm{n}=1}^{\mathrm{n}=\mathrm{i}} \Delta \mathrm{E}_{\mathrm{DLn}}
$$

and

$$
\mathrm{E}_{\mathrm{MCi}}=\sum_{\mathrm{n}=1}^{\mathrm{n}=\mathrm{i}} \Delta \mathrm{E}_{\mathrm{MCn}}
$$

Because this model is developed mainly for relatively thin and flexible woven fabric composites, the other possible energy absorbing mechanisms due to bending strain around the hinges at the edge of contact and at the edge of the cone, respectively, and in friction between projectile and yarn during the penetration process are not considered. In addition, energy absorbed in delamination and matrix cracking is not considered when projectile is penetrating flexible multi-layered textile targets. 
In the following, the main equations representing the present analytical model are arranged and compiled into a MATLAB program. The input data to the model are easily determined. The penetration process terminates when all fibers fail due to tension or all the layers fail due to shear plugging or due to the combined effect of both mechanisms. The projectile velocity at this moment represents its residual velocity. The model predictions are concerned with the determination of the ballistic resistance of a set of laminated textile and woven fabric composite targets when struck by a small caliber projectile, respectively.

\section{EXPERIMENTAL PROGRAM}

An experimental program has been conducted to study the penetration of a small caliber projectile into different laminated textile and woven fabric composite targets, respectively. Ballistic resistance for a set of targets against their penetration by $9 \mathrm{~mm}$ projectiles has been investigated. In general, the scheme of the experimental work included the following: (a) Target material choice and preparation, (b) Ballistic tests and measurements, (c) Postfiring examinations.

\section{Target Material Choice and Preparation}

In the present work, orthogonal two-dimensional woven fabric (plain weave), twaron and E-glass, were selected. They have low density, high tensile strength, good mechanical performance, corrosion resistance, and the construction of fabric enables spacing among the yarns which permits the resin to spread easily in-between the constructed fabric [13].

Target plates with dimensions of $200 \mathrm{~mm} \times 200 \mathrm{~mm}$, were prepared from six and nine layers of twaron and E-glass textiles, respectively. In addition, other targets consisting of twaron/epoxy and E-glass/epoxy composite targets with the same number of layers as textile targets were prepared. The hand lay-up contact molding method was selected for manufacturing the composite targets (twaron/Epoxy and E-glass/Epoxy) [14]. This method was used because: (i) it required minimum equipment, (ii) it could be used for small and large composites dimensions, and (iii) it produced smooth surfaces. The manufacturing process of composites was done using the facilities of the Chair of Material Science and Technology, M.T.C.

\section{Ballistic tests and measurements}

Ballistic tests were performed in order to determine the projectile impact and postperforation velocities for the different sets of prepared targets. Projectile arrival to the target was detected using velocity radar measuring system, Model 2605R. Measurements are based on the Doppler radar principle, which provides a signal proportional to the velocity of projectile. Departure of projectile from the target was detected using the velocity measuring device, Model HPI B571 optical target system. Time was measured and signals were processed to the B571-TR Time Recorder. This information was then transmitted to the PC where the projectile residual velocity could be displayed on its screen.

The ballistic experiments were performed in the ballistic shooing range, which had provisions for measurement of projectile impact and post-perforation velocities, 
respectively. As shown in Fig. 4., the ballistic set-up mainly consists of: ballistic rifle, impact velocity and post-perforation velocity measuring devices and target mount.

$9 \mathrm{~mm}$ projectiles having different impact velocities were fired against each prepared target; these velocities ranged from $200-400 \mathrm{~m} / \mathrm{s}$. The change in propellant charge mass was used to vary the projectile impact velocity. For each charge mass, a set of projectiles was fired against each tested target. Both the projectile impact and residual velocities, respectively, were measured using the velocity measuring systems used. To avoid the influence of plate boundaries on the perforation process, projectiles were fired at the effective area of each target surface which was far from its boundaries. The distance between two perforations on the same target surface was $60 \mathrm{~mm}$ at least. The tested targets are designated by symbols and digits as listed in Table 1.

\section{Post-Firing Examinations}

These were mainly concerned with the arrangement and the configurations of the set up, the projectile, and the target after its perforation. After each firing test, the test set up was examined to make sure that all connections were not damaged by the projectile or its fragments. Examining the laminated textiles and composite targets were done in order to determine the type of failure they exhibited. All interesting features related to target failure mode were photographed for subsequent analysis of test results.

\section{RESULTS AND DISCUSSIONS}

In the following, the present results are divided into: (i) ballistic firing test results, (ii) comparison between ballistic resistances of different tested targets, (iii) post-firing examinations of tested targets and recovered projectiles, and (iv) comparison between the obtained experimental measurements and the corresponding predictions of the present model.

\section{Ballistic Firing Test Results}

The ballistic test results due to the impact of different textiles and woven fabric composite targets by a $9 \mathrm{~mm}$ projectile having different impact velocities are presented. Both the projectile velocity drop ratio and energy loss ratio are chosen to represent the ballistic resistance of tested targets to penetration.

Figure 5 depicts the change of obtained residual velocity as a function of impact velocity for T-6, T-9, T/E-6 and T/E-9 targets, respectively. For each tested target, the present figure shows that the residual velocity increases with impact velocity in a quasi-linear manner over the range of impact velocity used. The difference between projectile residual velocities after perforating T-6 and T/E-6 targets is seen to diminish with the increase of impact velocity. In addition, both T-9 and T/E-9 targets are capable of trapping such a projectile at the lowest impact velocity used.

Figure 6 also plots the change of obtained residual velocity as a function of impact velocity for E-6, E-9, E/E-6 and E/E-9 targets, respectively. Similar to the measured results of the tested twaron textile and composite targets shown in Fig. 5, the present figure shows that the residual velocity for each $\mathrm{E}$-glass target increases with impact velocity in a quasi-linear 
manner. For each impact velocity, the present figure also shows the slight difference between the projectile residual velocities after perforating E-6 and E-9 targets.

Figure 7 plots the change of projectile velocity drop ratio DV/ $\mathrm{V}_{\mathrm{i}}\left(=\left(\mathrm{V}_{\mathrm{i}}-\mathrm{V}_{\mathrm{r}}\right) / \mathrm{V}_{\mathrm{i}}\right)$ with impact velocity for T-6, T-9, T/E-6 and T/E-9 targets, respectively. For each tested target, the present figure shows that the velocity drop ratio decreases with increasing impact velocity. The present figure also shows that the ballistic resistance of composite targets are slightly greater than that of textile targets having the same number of layers. In addition, the ballistic resistance of twaron textile and composite targets increases with increasing the number of their layers.

Figure 8 depicts the change of projectile energy loss ratio $D E / E_{i}\left(=\left(E_{i}-E_{r}\right) / E_{i}\right)$ with impact velocity for T-6, T/E-6, T-9 and T/E-9 targets, respectively. It is seen from the figure that the projectile consumed all of its initial kinetic energy when it impacted T-9 and T/E-9 targets at $V_{i}=198$ and $220 \mathrm{~m} / \mathrm{s}$, respectively. In addition, the projectile loses $70 \%$ of its initial kinetic energy in perforating a T-9 target at $V_{i}=375 \mathrm{~m} / \mathrm{s}$ and $75.6 \%$ of its initial kinetic energy in perforating a T/E-9 target at $V_{i}=379 \mathrm{~m} / \mathrm{s}$. This result shows that the used

Table 1. Composition snd designation of the tested targets.

\begin{tabular}{|c|c|c|c|c|c|}
\hline $\begin{array}{c}\text { Ser. } \\
\text { No. }\end{array}$ & $\begin{array}{c}\text { Target } \\
\text { composition }\end{array}$ & Designation & $\begin{array}{c}\text { Ser. } \\
\text { No. }\end{array}$ & Target composition & Designation \\
\hline 1 & Six layers twaron & T-6 & 5 & Six layers twaron/ Epoxy & T/E-6 \\
\hline 2 & $\begin{array}{c}\text { Nine layers } \\
\text { twaron }\end{array}$ & T-9 & 6 & $\begin{array}{c}\text { Nine layers twaron/ } \\
\text { Epoxy }\end{array}$ & T/E-9 \\
\hline 3 & Six layers E-glass & E-6 & 7 & Six layers E-glass/ Epoxy & E/E-6 \\
\hline 4 & $\begin{array}{c}\text { Nine layers E- } \\
\text { glass }\end{array}$ & E-9 & 8 & $\begin{array}{c}\text { Nine layers E-glass/ } \\
\text { Epoxy }\end{array}$ & E/E-9 \\
\hline
\end{tabular}

epoxy has a limited effect on increasing the ballistic resistance of twaron textile targets over the used impact velocity range.

Figure 9 plots the change of projectile velocity drop ratio $\mathrm{DV} / \mathrm{V}_{\mathrm{i}}$ with impact velocity for $\mathrm{E}-6$, $E-9, E / E-6$ and E/E-9 targets, respectively. For each target, the present figure shows that the velocity drop ratio has the same trend with impact velocity as that presented in Fig. 7 for twaron textile and its composite targets. In addition, the used epoxy has a significant effect on increasing the ballistic resistance of each E-glass textile target over the used impact velocity range.

Figure 10 plots the change of projectile energy loss ratio $D E / E_{i}$ with impact velocity for $E$ $6, E-9, E / E-6$ and E/E-9 targets, respectively. The present figure shows that the projectile loses $22.4 \%$ of its initial kinetic energy in perforating a T-6 target at $V_{i}=218 \mathrm{~m} / \mathrm{s}$ and $8.5 \%$ of its initial kinetic energy in perforating the same target at $V_{i}=370 \mathrm{~m} / \mathrm{s}$, whereas the projectile loses $43.9 \%$ of its initial kinetic energy in perforating a T/E-9 target at $V_{i}=207$ $\mathrm{m} / \mathrm{s}$ and $22.2 \%$ of its initial kinetic energy in perforating the same target at $V_{i}=365 \mathrm{~m} / \mathrm{s}$. Similar trends for increasing the ballistic resistance of T/E-9 targets compared with T-9 targets are obtained.

Figure 11 plots the change of projectile energy loss ratio $D E / E_{i}$ with impact velocity for $T-6$, T-9, E-6 and E-9 targets, respectively. It is seen from the figure that the ballistic resistance 
of E-glass and twaron textile targets increases with increasing their number of layers. In addition, the effect of increasing the number of layers of E-glass targets on their ballistic resistance diminishes with the increase of impact velocity. The measured results show that the projectile loses $70.1 \%$ of its initial kinetic energy in perforating a T-9 target at $\mathrm{V}_{\mathrm{i}}=$ $375 \mathrm{~m} / \mathrm{s}$, whereas the projectile loses $10.3 \%$ in perforating a $E-9$ target at $V_{i}=374 \mathrm{~m} / \mathrm{s}$. The obtained results recommend the twaron textile material to be used in constructing the body armors.

Figure 12 plots the change of projectile energy loss ratio $D E / E_{i}$ with impact velocity for T/E-6, T/E-9, E/E-6 and E/E-9 targets, respectively. Similar results for textile targets presented in Fig. 11 are obtained for their composite targets. In addition, the ballistic resistance of E-glass composite targets is so small compared with that of twaron composite targets having the same number of layers. The measured results show that the $\mathrm{T} / \mathrm{E}-9$ target traps the projectile at $\mathrm{V}_{\mathrm{i}}=198 \mathrm{~m} / \mathrm{s}$, whereas the projectile loses $53.8 \%$ of its initial kinetic energy in perforating an $E / E-9$ target at $V_{i}=209 \mathrm{~m} / \mathrm{s}$.

\section{Post-Firing Examinations of Recovered Projectiles and Tested Targets}

The recovered projectiles after perforating the tested textile and composite targets are inspected. These projectiles are subjected to considerable changes in their shapes. The degree of their deformations is mainly based on the resistance of the perforated target. Figure 13 shows a photograph for the back face of a T-6 tested target, whereas Fig. 14 shows a photograph for the back face of a T/E-6 target. It is seen from both figures that each target fails by tensile failure. The yarns of the fabric are subjected to high strains due to projectile penetration and the strains of the stretched yarns reach their failure value. The damage area during the penetration process of each target is localized. Moreover, the damage area in the back face of T-6 target is significantly greater than that of T/E- 6 composite target which is the main advantage of the used epoxy.

\section{Comparison between Experimental Measurements and Model Predictions}

In the following, results of the analytical model used to predict the projectile residual velocity after perforating each textile or composite target are presented. The data of projectile and each tested target are fed into the analytical model. Experimental measurements obtained in the present study are compared with the model predictions; good agreement is generally obtained. In addition, the maximum absolute relative difference between the measured residual velocity and the corresponding prediction of the analytical model is $15.5 \%$ when the projectile impacts a $E / E-9$ target at $V_{i}=209 \mathrm{~m} / \mathrm{s}$.

Figure 15 shows the predicted change of projectile residual velocity with impact velocity for T-6 and T-9 targets, respectively. For each textile target, the measured residual velocities corresponding to different impact velocities are also depicted on the same figure. Good agreement is generally found between measured and predicted residual velocities obtained by the analytical model over the used impact velocity range. Figure 16 also plots the measured and predicted change of projectile residual velocity with impact velocity for T/E-6 and T/E-9 targets, respectively, good agreement is generally found between measured and predicted residual velocities obtained by the analytical model over the used impact velocity range .

Figure 17 also plots the measured and the corresponding predicted change of projectile residual velocity with impact velocity for E-6 and E-9 targets, respectively. Good 
agreement is generally found between measured and predicted residual velocities obtained by the analytical model over the used range of impact velocity. In addition, Figure 18 shows good agreement between the measured and predicted residual velocities over the used impact velocity range for E/E-6 and E/E-9 targets, respectively.

\section{CONCLUSIONS}

The following conclusions could be drawn from the present work:

- The ballistic resistances of twaron textile and composite targets are relatively greater than that the corresponding E-glass targets having the same number of layers.

- Increasing the number of layers of E-glass targets has a limited effect on increasing their balistic resistance. Moreover, the used epoxy has a great effect on increasing their ballistic resistance.

- Post-firing examinations show that: (i) the projectile is subjected to a considerable deformation when perforating the tested targets; the degree of deformation depends on target resistance, (ii) yarns of twaron and E-glass textiles and their composite targets failed by tension, (iii) the damaged areas in E-glass and twaron composite targets are localized.

- For each tested target, the projectile residual velocities are compared with those of the corresponding predictions of the present analytical model; good agreement is generally obtained. Moreover, the maximum relative difference between the measured and predicted residual velocities is found to be $15.5 \%$ when a $9 \mathrm{~mm}$ projectile impacts $\mathrm{E} / \mathrm{E}$ 9 target at $\mathrm{V}_{\mathrm{i}}=209 \mathrm{~m} / \mathrm{s}$.

- Further analytical investigation is needed to consider the energy loss due to projectile deformation in the present model.

\section{REFERENCES}

1. N.K. Naik, P. Shrirao, B.C.K. Reddy,"Ballistic Impact Behaviour of Woven Fabric Composites: Formulation", Int. J. Impact Eng.,Vol. 32, No. 9, pp. 1521-1552 (2006).

2. S.S. Morye, P.J. Hine, R.A. Duckett, D.J. Carr and I.M. Ward, "Modelling of the Energy Absorption by Polymer Composites upon Ballistic Impact", Personal Armor System Sympo. [PASS 98], Colchester, U.K. (1998).

3. J.R. Vinson and J.A. Zukas,"On the Ballistic Impact of Textile Armor", J. Appl. Mech., pp. 263-268 (1975).

4. W.J. Taylor and J.R. Vinson, " Modeling Ballistic Impact Into Flexible Materials", AIAA J., Vol. 28, No. 12, pp. 2098-2103 (1989).

5. G. Zhu, W. Goldsmith and C.K.H. Dharan, "Penetration of Laminated Kevlar by Projectiles. II. Analytical Model", Int. J. Solids Sturctures, Vol. 29, No.4, pp. 421-436 (1992).

6. Bohong Gu, "Analytical Modeling for the Ballistic Perforation of Plannar PlainWoven Fabric Target by Projectile", Composites, Part B 34, pp. 361-371 (2003).

7. S.K.G. Castillo, S.S. Saez, J.L. Puente, E. Barbero, C. Navarro," Impact Behaviour of Preloaded Glass/Polyester Woven Plates", J. Comp. Sci. and Tech. (2008).

8. J.C. Smith, F.L. McCrackin and H.F. Scniefer," Stress-Strain Relationships in Yarns Subjected to Rapid Impact Loading, Part V. Wave Propagation in Long Textile Yarns Impacted Transversely", Text. Res. J., Vol. 28,No. 4, pp. 288-302 (1958).

9. D. Roylance, A. Wilde and G. Tocci, "Ballistic Impact of Textile Structures", Textile Research J., pp.34-41 (1973).

10. I.S.C. Benloulo, J. Rodriguez and V.S. Galvez, "A Simple Analytical Model to Simulate Textile Fabric Ballistic Impact Behavior", Textile Res. J. (1996). 
11. N.K.Naik, P. Shrirao and K.S. Reddy,"Baslllistic Impact Behavior of Woven Fabric Composites: Parameteric Studies", J. Mat. Sci. and Engng., Vol. A 412, pp. 04-116 (2005).

12. N.K. Naik and K.S. Reddy,"Delaminated Woven Fabric Composite Plates Under Transverse Quasi-Static Loading: Experimental Studies", J. Reinf. Plast Comp. Vol. 21, pp. 869-877 (2002).

13. A.R. Scott "Textiles for protection", Woodhead Publisishing limited and CRC Press L.L.C., USA (2005).

14. P.K. Mallic, 'Composite Engineering Hand Book', Marcel Dekker, Inc., USA (1991).

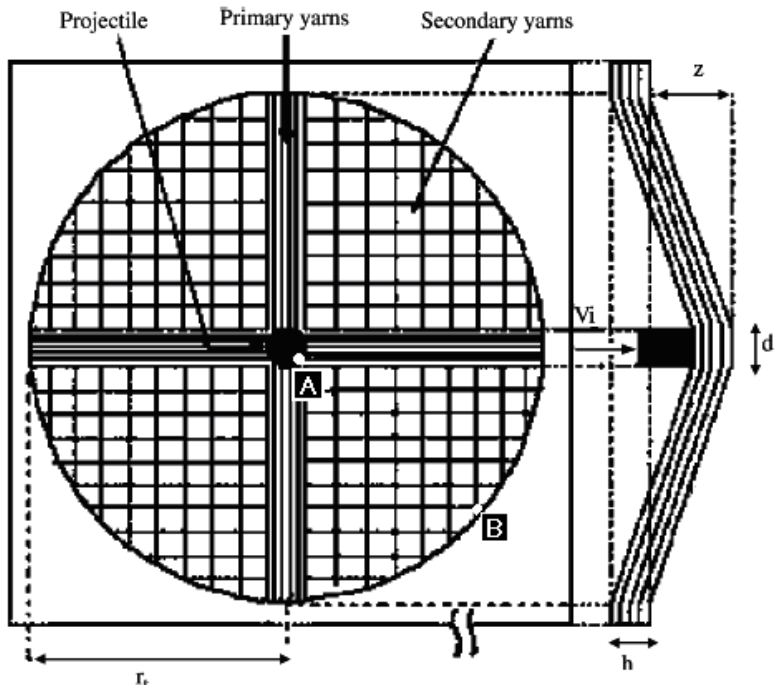

Fig.1. Cone formation during ballistic impact on the back face of the target [1].

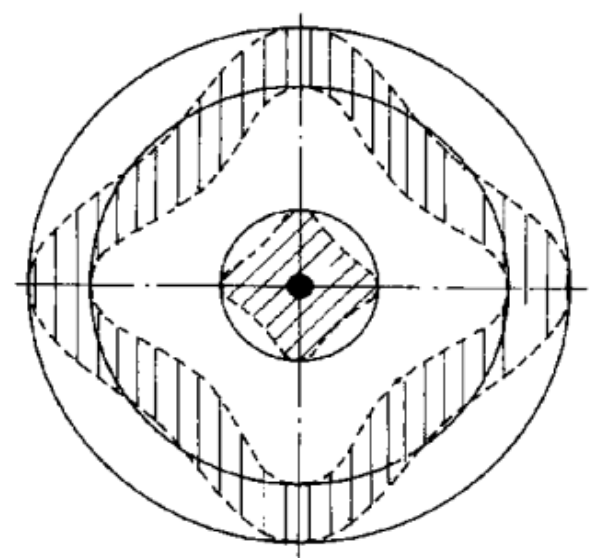

Fig.3. Experimentally observed shape of delaminated region [12].

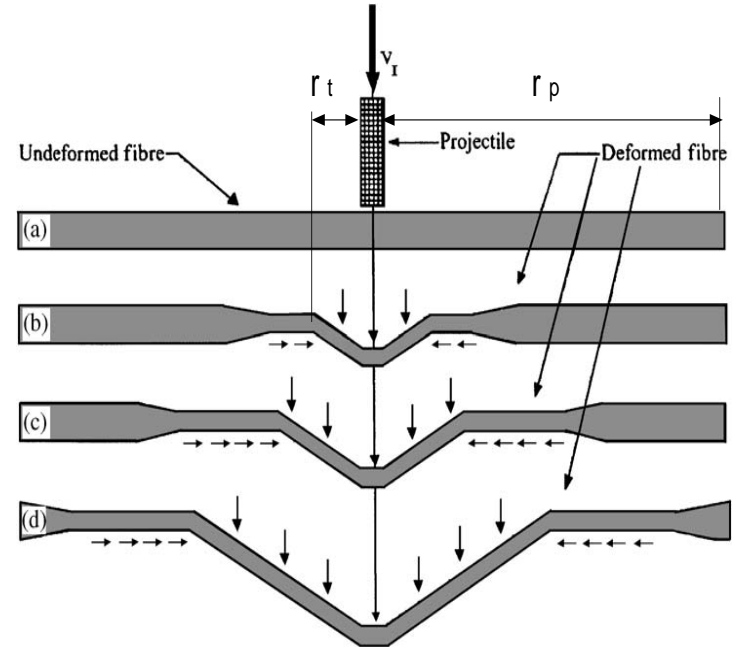

Fig.2. Configuration of a yarn/fiber before and after transverse impact: (a) before impact; (b)-(d) after impact [1].

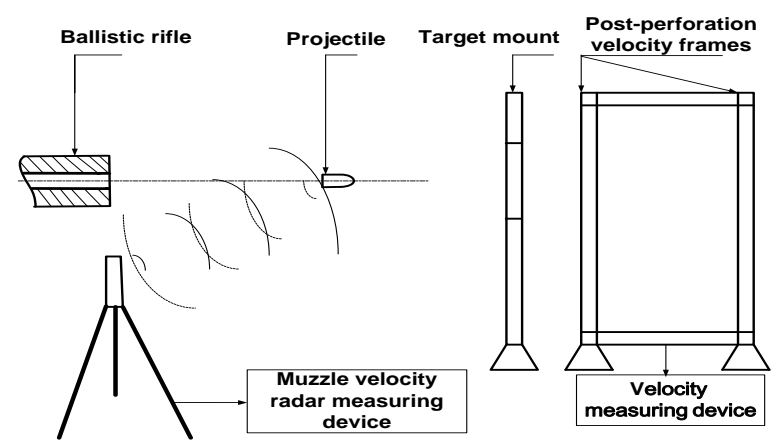

Fig.4. Scheme of ballistic set-up. 


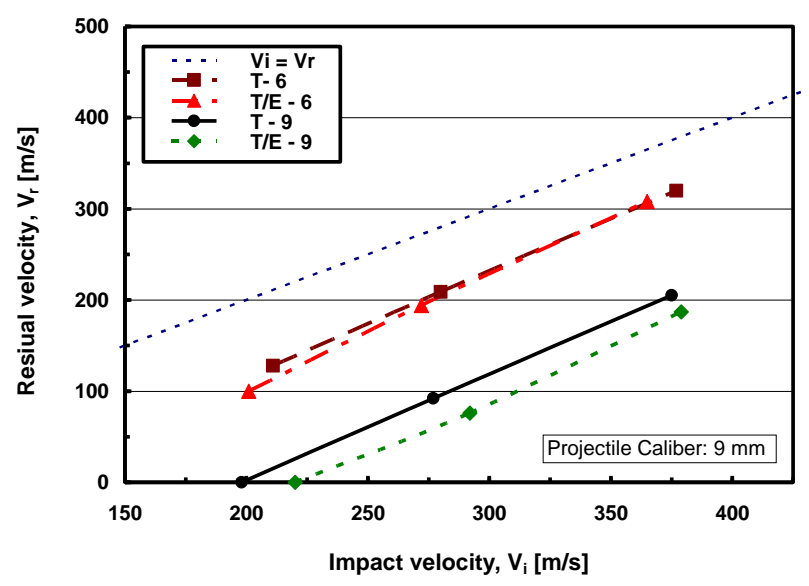

Fig.5. Change of measured projectile residual velocity with impact velocity for T-6, T-9, T/E-6 and T/E-9 targets, respectively.

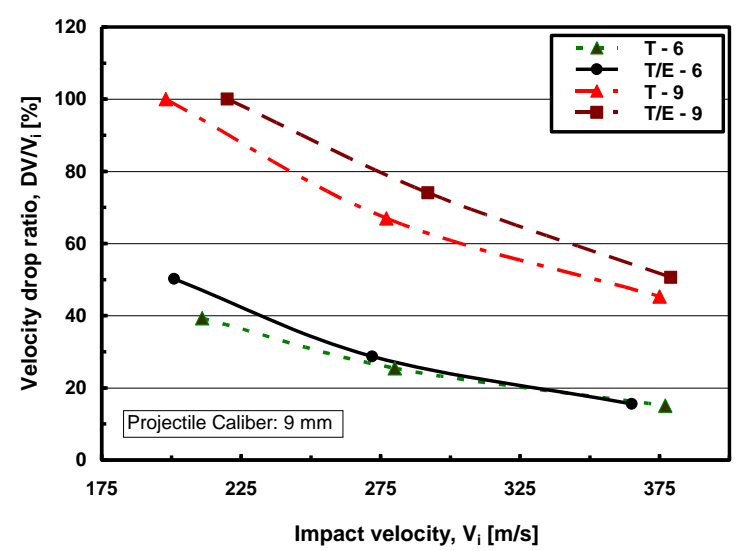

Fig.7. Change of measured velocity drop ratio with impact velocity for T-6, T-9, T/E-6 and T/E-9 targets, respectively.

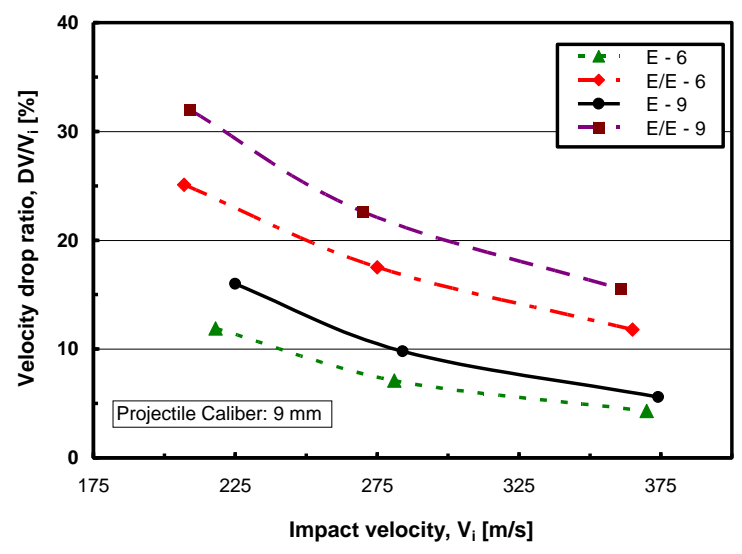

Fig.9. Change of measured velocity drop ratio with impact velocity for E-6, E9, and E/E-6 and E/E-9 targets, respectively.

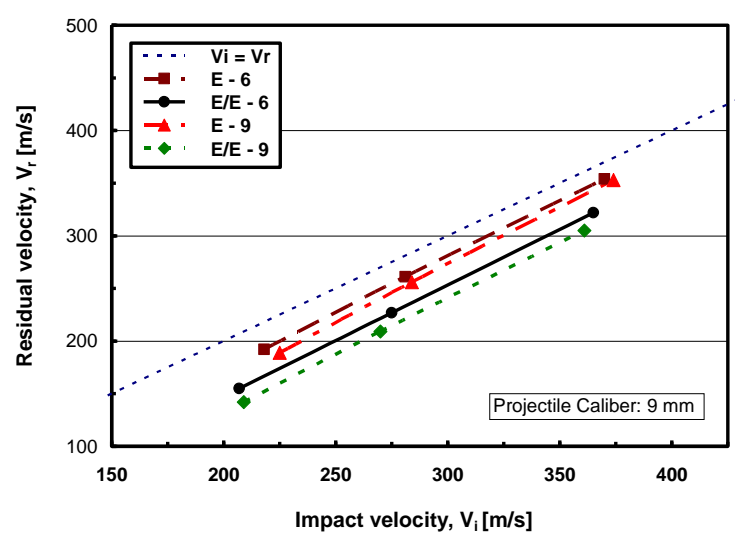

Fig.6. Change of measured projectile residual velocity with impact velocity for E-6 and E-9, E/E-6 and E/E-9 targets, respectively.

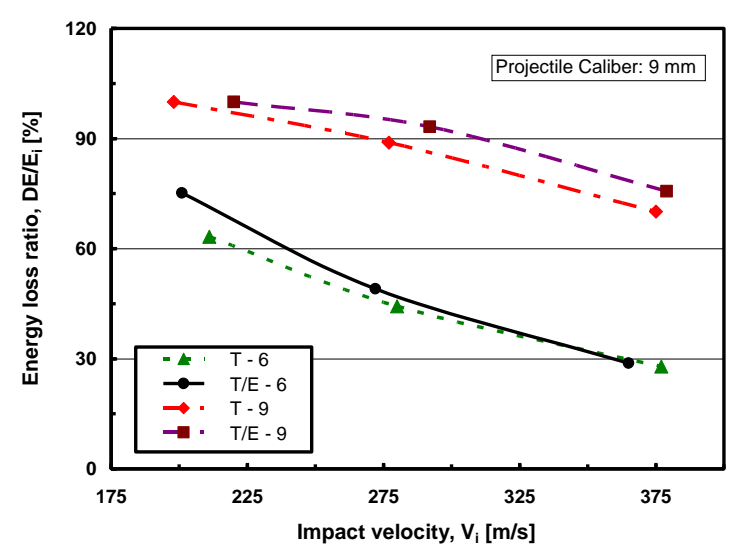

Fig.8. Change of measured energy loss ratio with projectile impact energy for $\mathrm{T}-6, \mathrm{~T}-9, \mathrm{~T} / \mathrm{E}-6$ and T/E-9 targets, respectively.

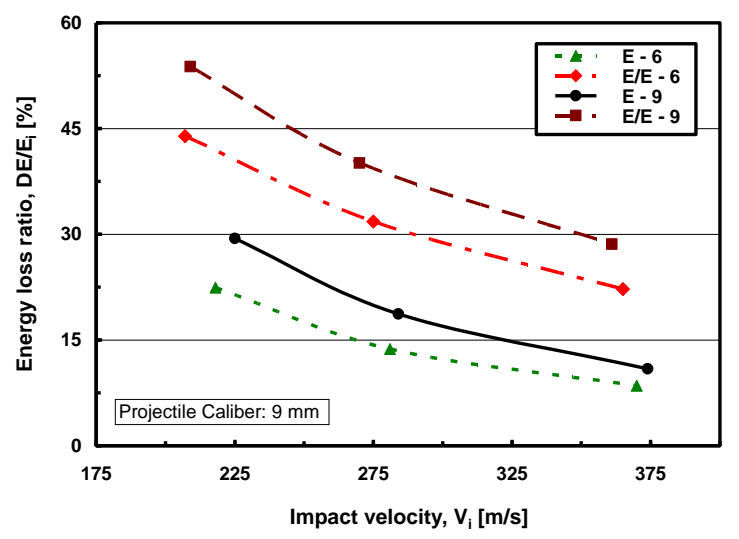

Fig.10. Change of measured energy loss ratio with impact energy for E-6, E-9, E/E-6 and E/E-9 targets, respectively. 


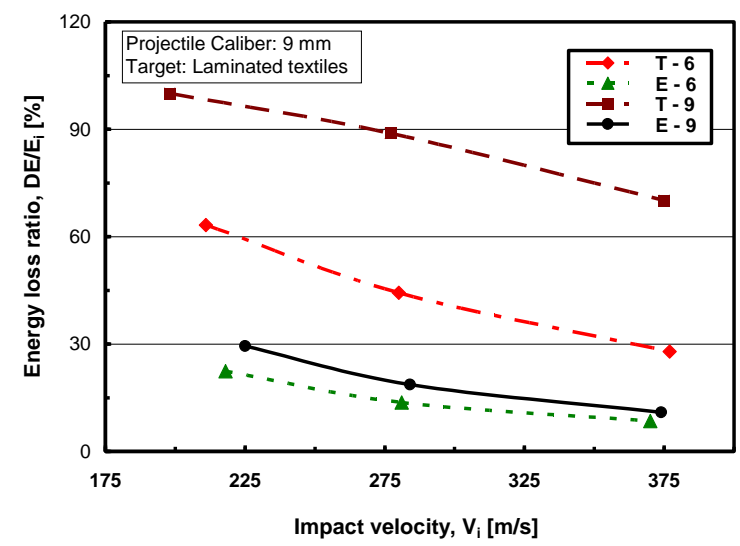

Fig.11. Change of measured energy loss ratio with impact energy for twaron and $\mathrm{E}$-glass textile targets, respectively.

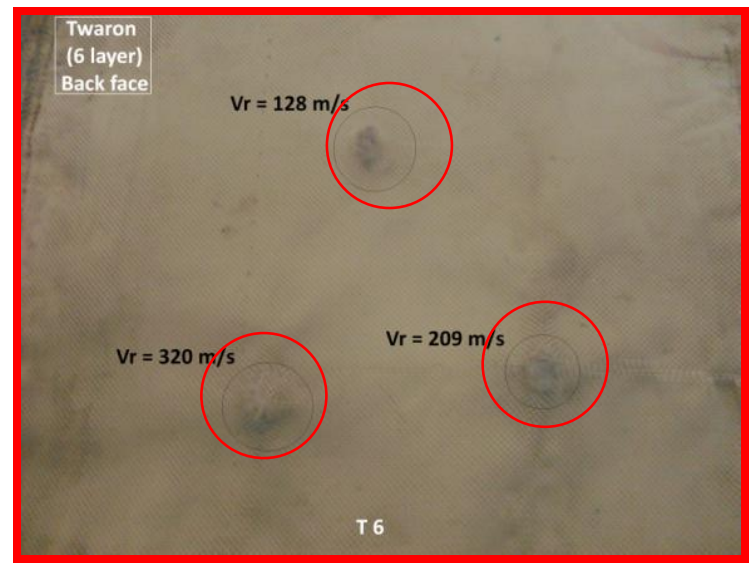

Fig. 13. A twaron textile target with 6 layers failed by tensile failure of yarns

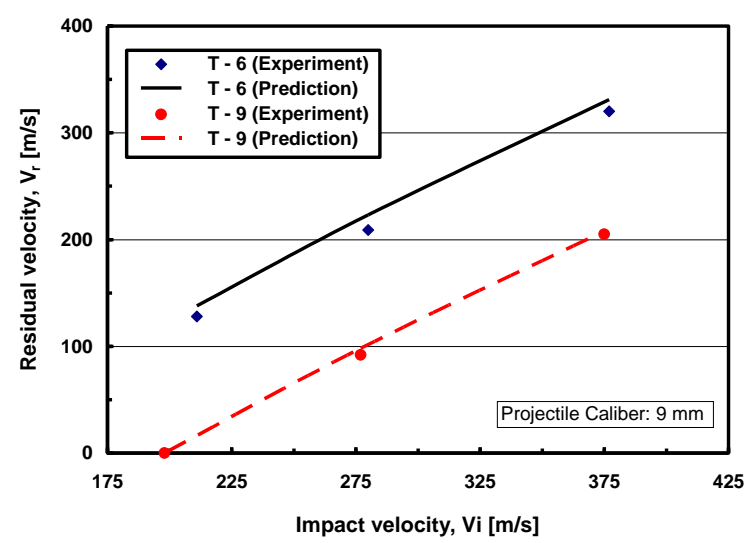

Fig. 15. Comparison between predicted and measured residual velocity versus impact velocity for T-6 and T-9 targets, respectively.

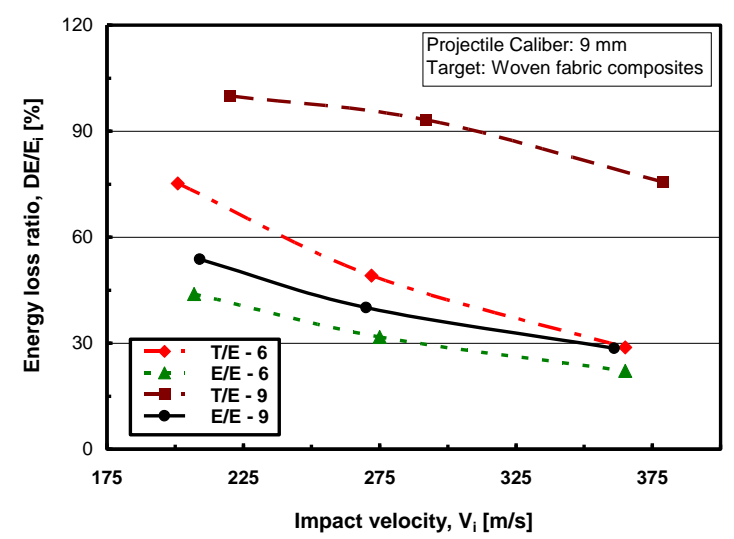

Fig.12. Change of measured energy loss ratio with impact energy for twaron and E-glass composite targets, respectively.

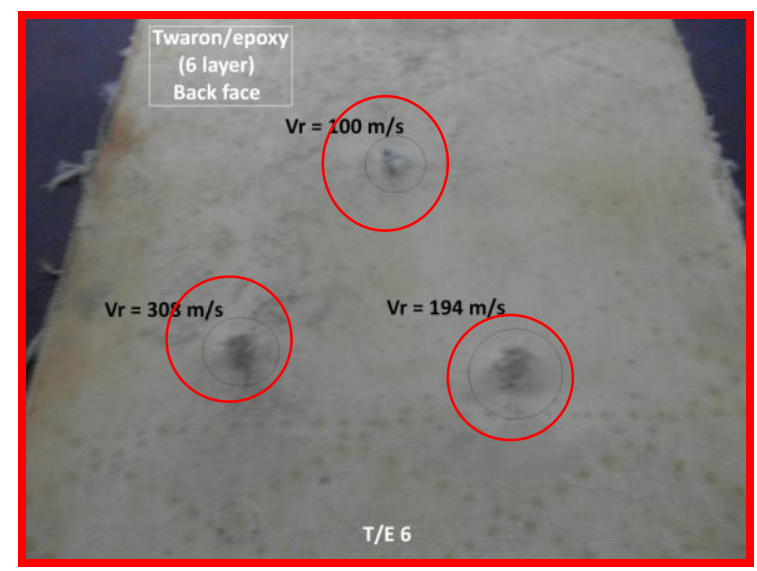

Fig. 14. A twaron composite target with 6 layers failed by tensile failure of yarns

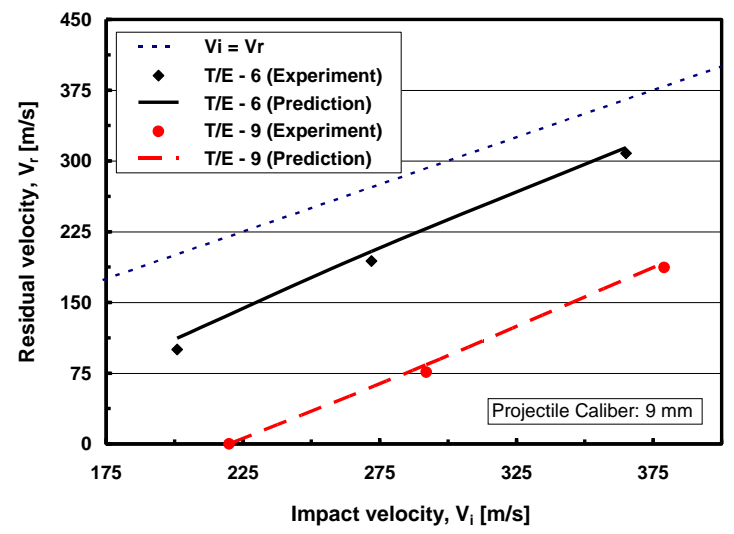

Fig. 16. Comparison between predicted and measured residual velocity versus impact velocity for T/E-6 and T/E-9 targets, 
respectively.

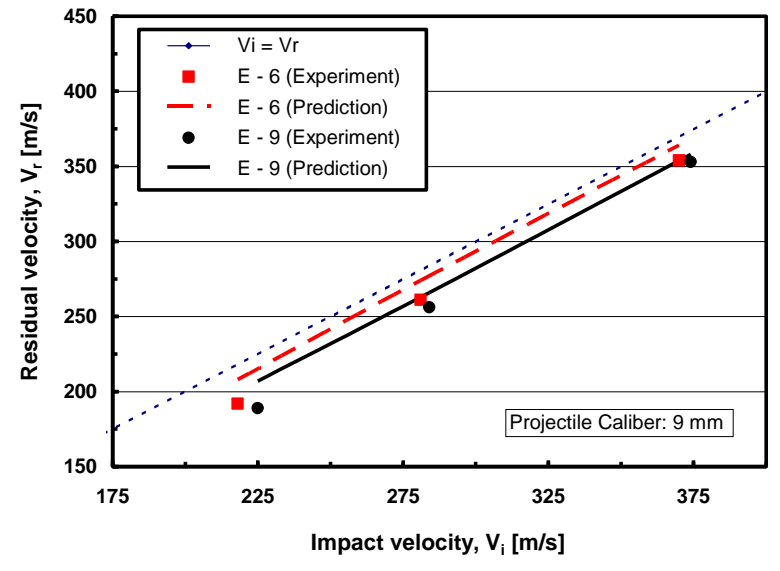

Fig. 17. Comparison between predicted and measured residual velocity versus impact velocity for E-6 and E-9 targets, respectively.

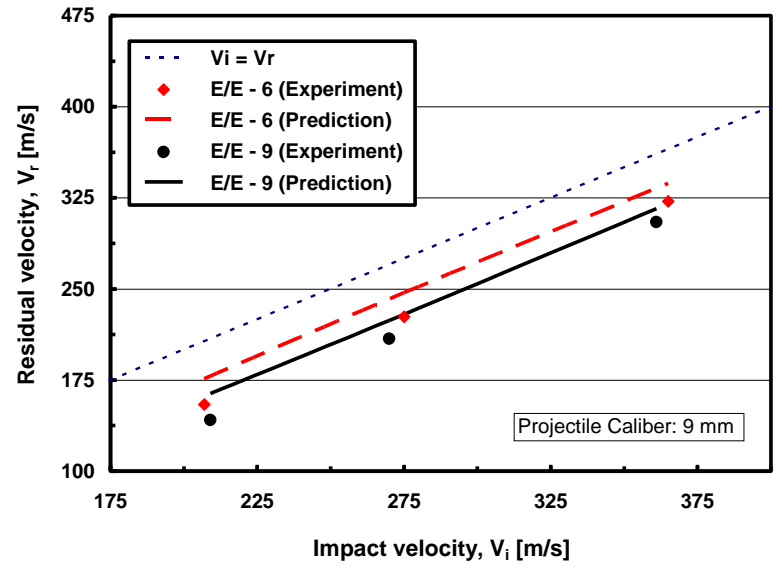

Fig. 18. Comparison between predicted and measured residual velocity versus impact velocity for $\quad E / E$ 6 and E/E-9 targets, respectively 